\section{Design of Near Perfect Reconstruction Oversampled Filter Banks for Subband Adaptive Filters}

Moritz Harteneck, Stephan Weiss, and Robert W. Stewart

\begin{abstract}
In this brief, a design algorithm for real-valued and complexvalued oversampled filter banks which yield a low level of inband alias and enable simple subband adaptive structures is presented. The filter banks are either based on complex modulation of a real-valued low-pass prototype or on the direct or modulated setups of real-valued filter banks. If real-valued filter banks are required, then the different channels will have different subsampling ratios so that the bandpass sampling theorem is not violated. This brief also presents design examples of real-valued and complex-valued filter banks.
\end{abstract}

Index Terms-Adaptive filtering, filter bank design, oversampled nearperfect reconstruction filter banks.

\section{INTRODUCTION}

Over the last two decades, adaptive filtering has been of considerable interest for many applications, such as acoustic echo cancelation [1], noise reduction, equalization, and beamforming [2]. Often, the adaptive system has to "model" a long-duration impulse response, as in the case of, e.g., the identification of room acoustics [3], [4]. Hence, with the high number of adaptive filter weights required, popular adaptive algorithms based on least-mean-squares and least squares techniques [5] become very computationally complex and exhibit a slow convergence.

One possibility to combat these problems is the use of adaptive algorithms, together with multirate techniques, to split the fullband problem into smaller subband problems. If the well-researched critically sampled perfect reconstruction filter banks are used to decompose the input signals, the subband signals are contaminated by aliasing, which requires adaptive filters in between adjacent bands to compensate for this distortion [6]. Thus, multichannel adaptive algorithms are being used in the subbands which exhibit a higher computational complexity and a slower convergence for correlated input signals. If the perfect reconstruction condition is dropped, critically sampled structures are obtainable, which do not need crossadaptive filters, as spectral gaps ensure that aliasing in the subbands does not occur [7], [8]. This spectral loss however prohibits exact system identification and can significantly distort signals passing through the filter bank. Applications in data communications or high-quality audio can be very sensitive to such errors.

Another possibility to achieve the decomposition of the input signals is to use oversampled filter banks (OSFB's), i.e., filter banks which generate redundancy in the subbands. One class of OSFB's is obtained via complex modulation from a real-valued low-pass prototype filter. If the prototype filter possesses a high stopband attenuation, then aliasing in the subbands is sufficiently suppressed. The subband signals in this class of OSFB's are all subsampled by the same subsampling ratio $S$. This filter bank can be modified to perform a single-sideband (SSB) modulation-like filter bank, yielding real-valued subband signals [9]-[11]. The trick is to modulate the bandpass signals into the baseband prior to decimation,

Manuscript received May 4, 1998; revised February 10, 1999. This paper was recommended by Associate Editor P. S. R. Diniz.

The authors are with the Signal Processing Division, Department of Electrical and Electronic Engineering, University of Strathclyde, Glasgow G1 1XW, Scotland, U.K.

Publisher Item Identifier S 1057-7130(99)06537-4.

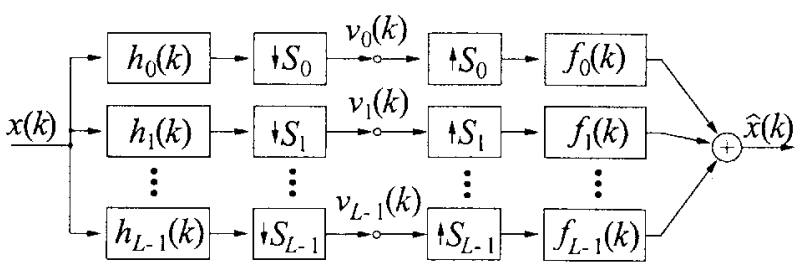

Fig. 1. General filter bank structure consisting of analysis and synthesis stages.

thus avoiding problems with bandpass sampling [12]. Since the prototype design is identical to the design for complex modulated filter banks, it is not discussed further in this paper. Another OSFB class consists of real-valued nonuniform filter banks [13] which have different subsampling ratios $S_{l}$ in each channel to satisfy the bandpass sampling theorem and avoid inband aliasing. In this case, the analysis filters posses different bandwidths which might be advantageous in, e.g., psychoacoustic coding [14].

In this paper, we present a fast-converging and efficient design algorithm for OSFB's with near-perfect reconstruction property which suppresses aliasing in the subbands, and is thus well suited for subband adaptive filtering. Therefore, Section II, will introduce two classes of real- and complex-valued OSFB's. Section III discusses an iterative least-squares design algorithm, for which examples will be shown in Section IV. Our notation uses a normalized sampling frequency $f_{s}=2$. Lower-case italic letters denote scalar values, lower- and upper-case boldface letters represent vector and matrix quantities, respectively, and $k$ is the discrete time index.

\section{TyPeS OF Filter BANKS}

Fig. 1 shows the concatenation of an analysis and a synthesis bank. On the analysis side, the input signal $x(k)$ is split into $L$ frequency bands by analysis filters $h_{l}(k)$ and decimated by a factor of $S_{l}$ in the $l$ th branch, giving the subband signal $v_{l}(k)$. On the synthesis side, each corresponding branch, consisting of an upsampler and a synthesis filter $f_{l}(k)$, restores the original fullband sampling rate and interpolates the subband signal. Summing over all branches then forms the output signal $\hat{x}(k)$. Such a filter bank possesses perfect or near perfect reconstruction property, if the output signal $\hat{x}(k)$ is essentially a copy of the input signal $x(k)$ delayed by $\Delta$ samples, i.e., $\hat{x}(k) \approx x(k-\Delta)$. Writing the transfer function of the filter bank shown in Fig. 1 in the frequency domain gives

$$
\hat{X}(z)=\sum_{l=0}^{L-1} \sum_{m=0}^{S_{l}-1} \underbrace{\frac{1}{S_{l}} F_{l}(z) H_{l}\left(z W_{S_{l}}^{m}\right)}_{T_{l, m}(z)} X\left(z W_{S_{l}}^{m}\right)
$$

where $\hat{X}(z), H_{l}(z), F_{l}(z)$, and $X(z)$ are the $z$-transforms of $\hat{x}(k)$, $h_{l}(k), f_{l}(k)$, and $x(k)$, respectively, and $W_{S}$ is a modulation factor $e^{-\jmath(2 \pi / S)}$. The transfer functions $T_{l, m}(z)$ describe the transfer of the different aliasing terms $m$ of each channel $l$. For the adaptive filters to work in the subbands without the need for information from adjacent subbands [6], the aliasing terms for $m>0$ have to be approximately zero, i.e.,

$$
T_{l, m}(z) \approx 0, \quad \forall l=0, \cdots, L-1, \quad m=1, \cdots, S_{l} .
$$




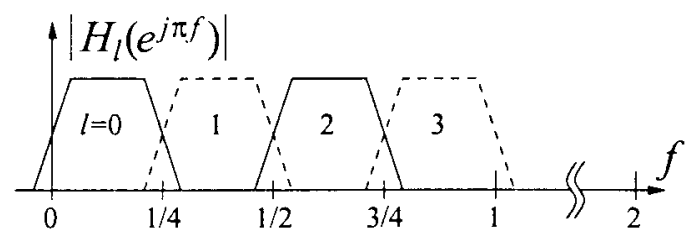

Fig. 2. Analysis filter setup for a four-channel complex-valued OSFB.

Note that if (2) is satisfied, (1) simplifies to

$$
\hat{X}(z)=\sum_{l=0}^{L-1} \frac{1}{S_{l}} F_{l}(z) H_{l}(z) X(z)
$$

i.e., perfect reconstruction and power complementarity become equivalent claims. To minimize the alias level $T_{l, m}(z)$ in the subband signals, complex- and real-valued setups are possible. These setups are outlined in Section II-A and II-B, respectively.

\section{A. Complex-Valued Filter Banks}

One way to implement an OSFB where inband aliasing is reduced to the level of the stopband attenuation of the analysis filters is to use a complex-valued set of analysis (see Fig. 2) and synthesis filters, whereby the filters are derived from a real-valued linear phase prototype $p(k)$ by a generalized discrete Fourier transform (GDFT) [10]

$$
h_{l}(k)=p(k) \cdot \exp \left[\jmath \frac{2 \pi}{L}\left(l+\frac{1}{2}\right)\left(k+k_{0}\right)\right] .
$$

A time offset $k_{0}=-\left(l_{p}-1\right) / 2$ in (4) ensures linear-phase analysis and synthesis filters if the prototype filter $p(k)$ is linear phase; its length $l_{p}$ is assumed to be even throughout this paper. Furthermore, this choice of $k_{0}$ causes real and imaginary parts of the analysis filters to separately fulfill linear-phase conditions. With the usual selection as complex-conjugate and time-reversed versions of the analysis filter, the synthesis filter take on a particularly simple form

$$
f_{l}(k)=\tilde{h}_{l}(k)=h_{l}(k) \text {. }
$$

The offset $1 / 2$ on the band index $l$ affects the position of the passbands of the $H_{l}(z)$ [10] and assures that the analysis filters are lined up equally in the frequency interval $f \in[0 ; 1]$, as shown in Fig. 2. For real-valued input $x(k)$, only the frequency bands from zero to one have to be covered by the analysis bank, since the remaining subbands are complex conjugate copies and therefore redundant. The oversampling ratio (OSR) for this filter bank equates to $(2 L) / S$, where $S$ is the common subsampling ratio for all channels. Note that to avoid aliasing in the subbands, the prototype filter $p(k)$ has to be a real-valued low-pass filter strictly limited to the frequency range $[-1 / S ; 1 / S]$. This type of OSFB approximates an implementation of Weyl-Heisenberg frames which are studied in detail in [15].

Collecting the coefficients of the prototype $p(k)$ and the analysis and synthesis filters $h_{l}(k)$ and $f_{l}(k)$ in vectors $\mathbf{p}, \mathbf{h}_{l}$ and $\mathbf{f}_{l}$

$$
\begin{aligned}
\mathbf{p} & =\left[\begin{array}{llll}
p(0) & p(1) & \cdots & p\left(l_{p}-1\right)
\end{array}\right]^{T} \\
\mathbf{h}_{l} & =\mathbf{f}_{l}=\left[\begin{array}{llll}
h_{l}(0) & h_{l}(1) & \cdots & h_{l}\left(l_{p}-1\right)
\end{array}\right]^{T}
\end{aligned}
$$

where superscript $T$ denotes transpose, and the modulation in (4) can be expressed by a convenient matrix-vector notation

$$
\mathbf{h}_{l}=\mathbf{f}_{l}=\mathbf{M}_{l} \cdot \mathbf{p}
$$

The matrices $\mathbf{M}_{l}$ are diagonal matrices with modulation factors (c.f. (4)) on their main diagonal. For further details on this type of filter bank and efficient polyphase implementations, refer to [10], [16], [11].

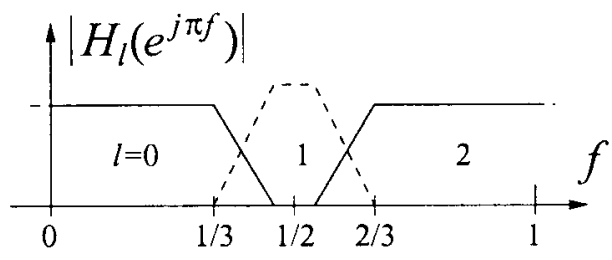

(a)

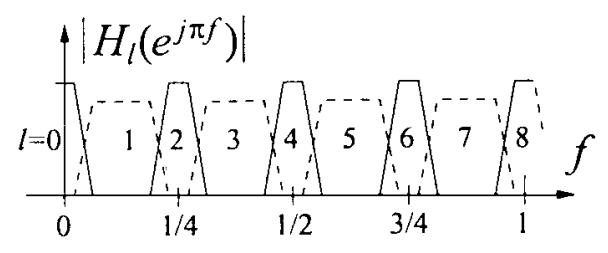

(b)

Fig. 3. Analysis filter setup for real-valued OSFB's using (a) three channels and (b) nine channels.

\section{B. Real-Valued Filter Banks}

Another way to obtain a filter bank satisfying (2) is to use a realvalued nonuniform OSFB and choose a setup where the bandpass sampling theorem is not violated [12], [13]. For a real-valued signal in the $l$ th channel to be undistorted due to decimation by a factor $S_{l}$, an interpretation of the bandpass sampling theorem requires the undecimated subband signal's band to lie entirely between any two consecutive frequency points among $f=i \cdot 1 / S_{l}, i=1,2, \cdots, S_{l}-1$.

Extending this to OSFB's means that every analysis filter $h_{l}(k)$ has to be placed in the frequency domain, such that the resulting signal does not violate the bandpass sampling theorem when subsampled by a factor $S_{l}$ and that the analysis filters have to be placed such that all frequencies are covered by at least one filter in order to allow reconstruction.

Fig. 3(a) shows the simplest setup of analysis filters of a nonuniform real-valued OSFB. In this filter bank, the low-pass and high-pass channels, produced by $H_{0}(z)$ and $H_{2}(z)$, are subsequently decimated by two and therefore are alias-free. The bandpass channel $H_{1}(z)$ is subsampled by a factor of three and also remains undistorted. Hence, the OSR for this filter bank equates to $\sum_{l=0}^{2} \cdot\left(1 / S_{l}\right)=133 \%$.

Another possibility to obtain real-valued OSFB's is to start from a cosine-modulated filter bank with $L_{0}$ channels, where the bandwidth of the analysis filters is reduced such that no aliasing occurs during decimation, and to fill the resulting spectral gaps by additional analysis filters which are derived from a second prototype to allow reconstruction. Filling the gaps with additional channels leads to a filter bank with a total of $L=2 L_{0}+1$ channels. All filters are derived from the two prototypes $p_{0}(k)$ and $p_{1}(k)$, as shown in Table I.

Fig. 3(b) shows, as an example, a nine-channel filter bank which is modulated according to Table I. Channels 1, 3, 5, and 7 are derived from the prototype $p_{0}(k)$ and can be subsampled by 4 . To avoid aliasing, the prototype filter $p_{0}(k)$ has to have a bandwidth of less than $1 / 8$, i.e., be limited to the frequency range $f \in[-1 / 8 ; 1 / 8]$. Channels $0,2,4,6$, and 8 , however, are derived from the prototype $p_{1}(k)$ and are subsampled by $8,6,5,6$, and 8 , respectively. These decimation ratios are chosen such that the allowed bandwidth of the prototype $p_{1}(k)$ is as large as possible and results in this example in the passband of the prototype to be restricted to the region $[-0.0833 ; 0.0833]$. The OSR of this filter bank is $178 \%$.

Analogous to Section II-A, the modulation can be expressed in matrix notation. More information on this type of filter bank can be found in [13], [17]-[19]. 
TABLE I

MOdULATION RULES

\begin{tabular}{l|l}
\hline band $l$ & analysis filter $h_{l}(k)$ \\
\hline 0 & $\sqrt{S_{0}} \cdot p_{1}(k)$ \\
$1,3, \ldots, 2 L_{0}-1$ & $2 \sqrt{S_{l}} \cdot p_{0}(k) \cdot \cos \left(k \cdot \frac{l}{2} \cdot \frac{\pi}{L_{0}}\right)$ \\
$2,4, \ldots, 2 L_{0}-2$ & $2 \sqrt{S_{l}} \cdot p_{1}(k) \cdot \cos \left(k \cdot \frac{l}{2} \cdot \frac{\pi}{L_{0}}\right)$ \\
$2 L_{0}$ & $\sqrt{S_{2 L_{0}}} \cdot p_{1}(k) \cdot \cos (k \cdot \pi)$ \\
\hline
\end{tabular}

If a filter bank with a higher number of channels is required, the presented cosine modulation approach can be used, starting with a higher $L_{0}$, or different setups can be found which satisfy the bandpass sampling theorem. Alternatively, a previously designed filter bank can be iterated which, however, leads in general to suboptimal solutions.

\section{Design Algorithm}

A common choice in the design of filter banks is to choose the synthesis filters to be time-reversed and complex-conjugate versions of the analysis filters. This yields a reduction in the free design parameters. According to the theory of frames [20], this also leads to very close approximation of signal decompositions with a fixed energy translation between time and subband domain, regardless of the input signal.

To design OSFB's, as presented in Section II, an iterative leastsquares algorithm [22], [23] is used. The performance criterion to be minimized by the algorithm is a combination of the filter bank reconstruction error $\xi_{1}$ and the stopband energy $\xi_{2}$ of the analysis filters $h_{l}(k)$

$$
\xi=\xi_{1}+\gamma \cdot \xi_{2}
$$

where $\gamma$ is a positive weighting factor which trades off between the importance of the reconstruction error and the stopband attenuation. In the following, we discuss separately formulations for both $\xi_{1}$ and $\xi_{2}$, and finally, present the design algorithm in Section III-C.

\section{A. Reconstruction Error}

If inband aliasing is sufficiently suppressed, the impulse response $t(k)$ of the overall filter bank, as given in Fig. 1, is the time-domain formulation of (3), and can be written as a convolution of the analysis and the synthesis filters

$$
t(k)=\sum_{l=0}^{L-1} \frac{1}{S_{l}}\left(h_{l}(k) * f_{l}(k)\right)
$$

where "*" denotes convolution and the factor $1 / S_{l}$ takes account of the power loss in the channels due to decimation. Using matrix notation, the convolution in the $l$ th branch can be calculated by [24]

$$
\begin{aligned}
\mathbf{t}_{l} & =\left[\begin{array}{c}
t_{l}(0) \\
t_{l}(1) \\
t_{l}(2) \\
\vdots \\
t_{l}\left(2 l_{p}-2\right)
\end{array}\right] \\
& =\left[\begin{array}{cccc}
h_{l}(0) & 0 & \cdots & 0 \\
h_{l}(1) & h_{l}(0) & \cdots & 0 \\
h_{l}(2) & h_{l}(1) & \cdots & 0 \\
\vdots & \vdots & \ddots & \vdots \\
0 & 0 & \cdots & h_{l}\left(l_{p}-1\right)
\end{array}\right]\left[\begin{array}{c}
f_{l}(0) \\
f_{l}(1) \\
f_{l}(2) \\
\vdots \\
f_{l}\left(l_{p}-1\right)
\end{array}\right] \\
& =\mathbf{H}_{l} \cdot \mathbf{f}_{l} .
\end{aligned}
$$

Evaluating the summation of (9), the impulse response of the whole filter bank can be expressed as

$$
\mathbf{t}=\left[\begin{array}{llll}
\frac{1}{S_{0}} \mathbf{H}_{0} & \frac{1}{S_{1}} \mathbf{H}_{1} & \cdots & \frac{1}{S_{L-1}} \mathbf{H}_{L-1}
\end{array}\right]\left[\begin{array}{c}
\mathbf{f}_{0} \\
\mathbf{f}_{1} \\
\vdots \\
\mathbf{f}_{L-1}
\end{array}\right]=\mathbf{H} \cdot \mathbf{f} .
$$

To get a measure of the reconstruction error, the Euclidean distance between the impulse response $\mathbf{t}$ and a prefect delay (represented by the vector $\mathbf{v}$ ) is evaluated

$$
\xi_{1}=\|\mathbf{t}-\mathbf{v}\|^{2}=\|\mathbf{H} \cdot \mathbf{f}-\mathbf{v}\|^{2} .
$$

\section{B. Stopband Energy}

As described in [25], [22], a measure for the energy contained in the stopband of a linear phase analysis filter $h_{l}(k)$ can be calculated by using a dense grid of frequency points $\left\{\omega_{0}, \omega_{1}, \cdots, \omega_{N}\right\}$ covering the whole stopband and calculating

$$
\begin{aligned}
\xi_{2, l} & =\left\|\left[\begin{array}{cccc}
1 & \cos \left(\omega_{0} \cdot 1\right) & \cdots & \cos \left(\omega_{0} \cdot\left(l_{p}-1\right)\right) \\
1 & \cos \left(\omega_{1} \cdot 1\right) & \cdots & \cos \left(\omega_{1} \cdot\left(l_{p}-1\right)\right) \\
\vdots & \vdots & \ddots & \vdots \\
1 & \cos \left(\omega_{N} \cdot 1\right) & \cdots & \cos \left(\omega_{N} \cdot\left(l_{p}-1\right)\right)
\end{array}\right]\left[\begin{array}{c}
h_{l}(0) \\
h_{l}(1) \\
\vdots \\
h_{l}\left(l_{p}-1\right)
\end{array}\right]\right\|^{2} \\
& =\left\|\mathbf{P}_{l} \cdot \mathbf{h}_{l}\right\|^{2} .
\end{aligned}
$$

Here, the matrix $\mathbf{P}_{l}$ describes the required specifications on the stopbands of the $l$ th analysis filter.

\section{Minimization Algorithm}

Using the above definitions and rewriting (8) in a matrix vector notation for a general $L$-channel filter bank, and assuming analysis and synthesis filters to be the same, such that a tight frame is achieved in very close approximation, the performance criterion can be written as

$$
\xi=\left\|\left[\begin{array}{c}
\mathbf{H} \\
\gamma \mathbf{P}
\end{array}\right] \mathbf{h}-\left[\begin{array}{l}
\mathbf{v} \\
\mathbf{0}
\end{array}\right]\right\|^{2} .
$$

In (14), $\mathbf{P}=\operatorname{diag}\left(\mathbf{P}_{0}, \mathbf{P}_{1}, \cdots, \mathbf{P}_{L-1}\right)$ is a matrix describing the frequency specifications on all analysis filters and $\mathbf{h}=$ $\left[\begin{array}{llll}\mathbf{h}_{0}^{T} & \mathbf{h}_{1}^{T} & \cdots & \mathbf{h}_{L-1}^{T}\end{array}\right]^{T}$ is a collection of the analysis filters. As defined in (11) the matrix $\mathbf{H}$ consists of the matrices $\mathbf{H}_{l}$ and these matrices are dependent on the analysis filters $\mathbf{h}_{l}$. Therefore the performance criterion (14) is to the power of four dependent on the design parameters.

To enforce linear phase filters in the design algorithm, the axial symmetry of the filter impulse responses can be exploited. As an example, the prototype filter $p(k)$ is completely characterized by its first half of the impulse response. In matrix notation, this relationship can be expressed as

$$
\mathbf{p}=\mathbf{L} \cdot \overline{\mathbf{p}}
$$

where $\mathbf{L}$ is an $l_{p} \times\left(l_{p} / 2\right)$ matrix defined by $\mathbf{L}=\left[\begin{array}{ll}\mathbf{I}_{l_{p} / 2} & \mathbf{J}_{l_{p} / 2}\end{array}\right]^{T}, \mathbf{I}$ being an identity and $\mathbf{J}$ an inverse identity matrix. Incorporating (15) into the design effectively halves the degrees of freedom.

Thus, for the three-channel real-valued OSFB discussed in Section II-B, the performance criterion can now be written as

$$
\xi=\left\|\left[\begin{array}{ccc}
\frac{1}{S_{0}} \mathbf{H}_{0} \mathbf{L} & \frac{1}{S_{1}} \mathbf{H}_{1} \mathbf{L} & \frac{1}{S_{2}} \mathbf{H}_{2} \mathbf{L} \\
\gamma \mathbf{P}_{0} \mathbf{L} & \mathbf{0} & \mathbf{0} \\
\mathbf{0} & \gamma \mathbf{P}_{1} \mathbf{L} & \mathbf{0} \\
\mathbf{0} & \mathbf{0} & \gamma \mathbf{P}_{2} \mathbf{L}
\end{array}\right]\left[\begin{array}{l}
\overline{\mathbf{h}}_{0} \\
\overline{\mathbf{h}}_{1} \\
\overline{\mathbf{h}}_{2}
\end{array}\right]-\left[\begin{array}{l}
\mathbf{v} \\
\mathbf{0} \\
\mathbf{0} \\
\mathbf{0}
\end{array}\right]\right\|^{2}
$$

where the matrices $\mathbf{P}_{l}$ describes the stopband requirements to eliminate the aliasing caused by the subsampling process. 
TABLE II

Iterative LeAst-SQuares Design Algorithm

\begin{tabular}{c||l}
\hline Step & Instruction \\
\hline $1:$ & initialize filters $\mathbf{h}(i)$, set $i=1$ \\
2: & build $\mathbf{H}_{l}(i-1)$ from $\mathbf{h}_{l}(i-1)$ \\
$3:$ & minimize with respect to $\mathbf{h}(i)$ \\
& $\xi=\left\|\left[\begin{array}{c}\mathbf{H}(i-\mathbf{1}) \\
\gamma \mathbf{P}\end{array}\right] \mathbf{h}(i)-\left[\begin{array}{l}\mathbf{v} \\
0\end{array}\right]\right\|^{2}$ \\
4: & $\begin{array}{l}\text { apply relaxation } \\
\mathbf{h}(i)=\tau \mathbf{h}(i)+(1-\tau) \mathbf{h}(i-1)\end{array}$ \\
$5:$ & $\begin{array}{l}\text { check stop condition } \\
\text { if }\|\mathbf{h}(i)-\mathbf{h}(i-1)\|<\epsilon \text { then stop }\end{array}$ \\
$6:$ & $i=i+1$, goto 2: \\
\hline
\end{tabular}

For the nine channel real-valued OSFB as presented in Section II$\mathrm{B}$, which is derived from two prototypes $\mathbf{p}_{0}$ and $\mathbf{p}_{1}$, the performance criterion simplifies to

$$
\xi=\left\|\left[\begin{array}{cc}
\sum_{l \in S_{0}} \frac{1}{S_{l}} \mathbf{H}_{l} \mathbf{M}_{l} \mathbf{L} & \sum_{l \in S_{1}} \frac{1}{S_{l}} \mathbf{H}_{l} \mathbf{M}_{l} \mathbf{L} \\
\gamma \mathbf{P}_{0} \mathbf{L} & \mathbf{0} \\
\mathbf{0} & \gamma \mathbf{P}_{1} \mathbf{L}
\end{array}\right]\left[\begin{array}{c}
\overline{\mathbf{p}}_{0} \\
\overline{\mathbf{p}}_{1}
\end{array}\right]-\left[\begin{array}{l}
\mathbf{v} \\
\mathbf{0} \\
\mathbf{0}
\end{array}\right]\right\|^{2}
$$

where the matrices $\mathbf{P}_{0}$ and $\mathbf{P}_{1}$ describe the frequency requirements on the prototypes $\mathbf{p}_{0}$ and $\mathbf{p}_{1}$, respectively, and the set $S_{l}$ denotes the set of filters derived from the prototype $\mathbf{p}_{l}$.

The performance criterion for the complex-valued OSFB presented in Section II-A, which is derived from only one real-valued prototype $\mathbf{p}$, further simplifies to

$$
\xi=\|\left[\frac{1}{S} \sum_{l=0}^{L-1} \mathbf{H}_{l} \mathbf{M}_{l} \mathbf{L} \mathbf{P}=\overline{\mathbf{p}}-\left[\begin{array}{l}
\mathbf{v} \\
\mathbf{0}
\end{array}\right] \|^{2}\right.
$$

where $S$ is the common subsampling ratio and $\mathbf{P}$ describes the frequency requirements on the prototype.

To perform the minimization of (14), an iterative least squares technique [22], [26], [23] is used where the matrix $\mathbf{H}$, which is being built from the analysis filters, is substituted by the matrix being built from an old set of analysis filters. This linearization gives a quadratic approximation of the performance surface. To ensure convergence, a relaxation step is added to the algorithm. The design algorithm can now be formulated iteratively as shown in Table II, where $\tau$ is the relaxation parameter which has to be chosen between zero and one and $\epsilon$ quantifies the stop condition. The minimization of Step 2 can be conveniently performed by a QR decomposition [27].

\section{Design EXAmples}

In this section, design examples of OSFB's targeted for subband adaptive filtering are shown. The filter banks have been designed to yield a reconstruction error and an inband alias of about $-80 \mathrm{~dB}$, giving a resolution of approximately 13 bits. These specifications are targeted at audio applications, but higher quality designs are easily achievable with the presented algorithms [13]. The parameters settings in the design algorithm are $\tau=0.5$ and $\gamma=10$.

Fig. 4 shows the transfer functions of the analysis and synthesis filters of a four-channel OSFB. The filter bank is designed for aliasfree decimation by $S=6$ [11], giving an OSR of $133 \%$. To achieve the specifications, the design algorithm needs 14 iterations to produce the prototype filter with a required number of $l_{p}=192$ taps.

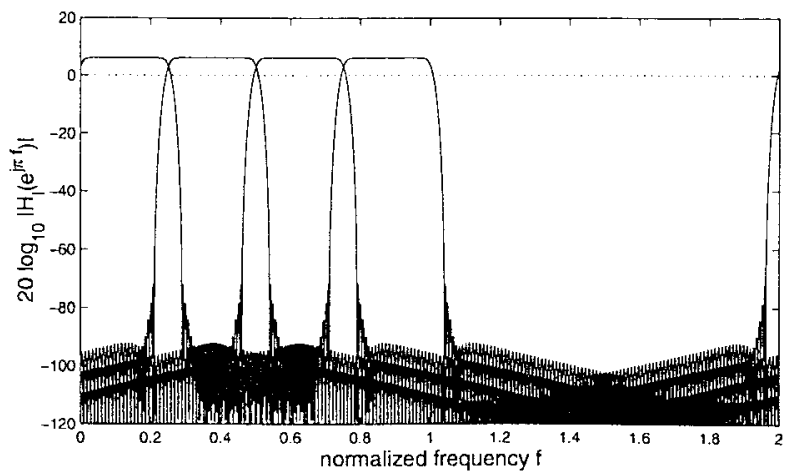

Fig. 4. Four-channel complex-valued OSFB.

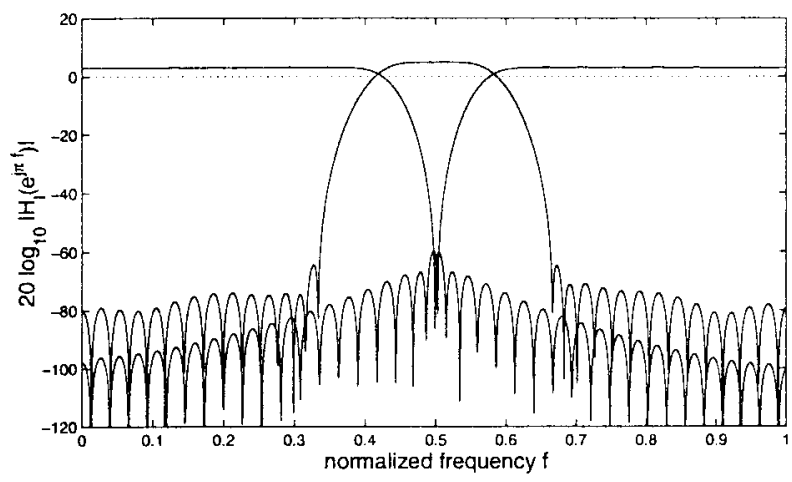

(a)

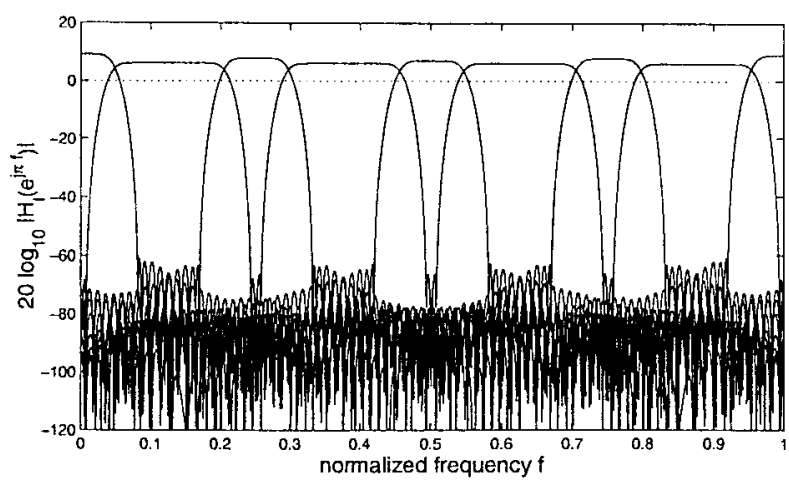

(b)

Fig. 5. Real-valued OSFB's using (a) three channels and (b) nine channels.

Fig. 5(a) shows the transfer functions of the analysis filters of the filter bank used to implement a three channel setup as presented in Section II-B. To avoid inband aliasing, the stopbands of the analysis filters $h_{l}(k)$ extend over $[0.495 \cdots 1],[0 \cdots 0.346] \cup[0.643 \cdots 1]$, and $[0 \cdots 0.505]$, which are subsampled by 2,3 , and 2 , respectively. Note that the stopbands of the prototypes have been enlarged slightly to create a guard band. To achieve the required specifications, the filters need 74 taps and the filter bank generates an OSR of $133 \%$. The design algorithm converged after 12 iterations.

Fig. 5(b) shows the transfer functions of the real-valued filters used in the analysis bank of the nine channel OSFB as presented in Section II-B. The stopband of the prototype $p_{0}(k)$, generating the channels $1,3,5$, and 7 , extends from 0.123 to 1 , and the stopband of the prototype $p_{1}(k)$, generating the other channels, extends from 0.083 to 1 . Note again that the stopbands have been slightly enlarged. To avoid aliasing, the channels are decimated from the left to the right by $8-4-6-4-5-4-6-4-8$, giving an OSR of $178 \%$. To meet 
the specifications, the analysis filters required 172 taps. The design algorithm needs 11 iterations to achieve the minimization.

\section{CONCLUSION}

In this paper, a design algorithm for OSFB's has been presented, which is designed for the implementation of subband adaptive filters. Therefore, two classes of filter banks, a real-valued and a complexvalued class, have been introduced, which satisfy the requirement of a low inband aliasing level, such that single-channel adaptive filters operating in each subband are possible. An algorithm for the design of these filter banks has been presented which is based on an iterative least squares technique to minimize the performance criterion. Finally design examples for the real-valued case and the complex-valued case have been given.

Using these filter banks, together with adaptive filters, significant computational savings are achievable. For example, using leastsquares adaptive filters, the subband approach with real-valued filter banks reduces the computational requirements to $28.5 \%$ and $8.5 \%$, compared with a fullband algorithm for the presented three channel and the nine channel OSFB's, respectively. Using the presented complex-valued filter bank, the structure needs about $7.4 \%$ of the computational complexity of a fullband implementation. These savings can be even more significant if the number of channels and the associated decimation factors are increased. Note that the given percentages refer to the cost of adaptive filtering only. However, the cost of filter bank computations can be kept low by judicious implementation using polyphase factorizations [15], [28].

Simulations using the filter banks, together with adaptive filters, which are not presented in this paper, have shown that the performance in terms of minimum mean-squared error is limited by the amount of inband aliasing and reconstruction error, which come from the used filter bank [29]. For colored-noise input signals, however, in general these subband structures have shown an improvement in terms of convergence speed.

\section{ACKNOWLEDGMENT}

The authors would like to thank J. McWhirter and I. Proudler of DERA, Malvern, U.K., for their support in this work, and J. M. Páez-Borrallo of the Universidad de Madrid for his contribution.

\section{REFERENCES}

[1] E. Hänsler, "The hands-free telephone problem: A second annotated bibliography update," in Proc. Int. Workshop Acoustic Echo and Noise Control, Roros, Norway, June 1995.

[2] L. C. Godara, "Application of the fast Fourier transform to broadband beamforming," J. Acoust. Soc. Amer., vol. 98, no. 1, pp. 230-240, July 1995.

[3] M. Schönle, N. J. Fliege, and U. Zölzer, "Parametric approximation of room impulse response by multirate systems," in Proc. IEEE ICASSP, 1993, vol. 1, pp. $153-156$

[4] N. J. Fliege, Multirate Digital Signal Processing. New York: Wiley, 1994.

[5] S. Haykin, Adaptive Filter Theory, 3rd ed. Englewood Cliffs, NJ: Prentice-Hall, 1996.

[6] A. Gilloire and M. Vetterli, "Adaptive filtering in subbands with critical sampling: Analysis, experiments, and application to acoustic echo cancellation," IEEE Trans. Signal Processing, vol. 40, pp. 1862-1875, Aug. 1992.

[7] Y. Yamada, H. Ochi, and K. Kiya, "A subband adaptive filter allowing maximally decimation," IEEE J. Select. Areas Commun., vol. 12, pp. 1548-1552, Sept. 1994.

[8] O. Tanrikulu, A. G. Constantinides, and J. A. Chambers, "Residual echo signal in critically sampled sub-band acoustic echo cancellers based on IIR and FIR filter banks," IEEE Trans. Signal Processing, vol. 45, pp. 901-912, Apr. 1997.
[9] G. Wackersreuther, "Some new aspects of filters for filter banks," Trans. Acoust., Speech, Signal Processing, vol. 34, pp. 1182-1200, Oct. 1986.

[10] R. E. Crochiere and L. R. Rabiner, Multirate Digital Signal Processing. Englewood Cliffs, NJ: Prentice-Hall, 1983.

[11] S. Weiss, L. Lampe, and R. W. Stewart, "Efficient implementations of complex and real valued filter banks for comparative subband processing with an application to adaptive filtering," in Proc. Int. Symp. Communication Systems \& Digital Signal Processing, Sheffield, U.K., Apr. 1998, vol. 1, pp. 32-35

[12] A. J. Coulson, "A generalization of nonuniform bandpass sampling," IEEE Trans. Signal Processing, vol. 43, pp. 694-704, 1995.

[13] M. Harteneck, J. M. Páez-Borallo, and R. W. Stewart, "A filter bank design for oversampled filter banks without aliasing in the subbands," Electron. Lett., vol. 33, no. 18, pp. 1538-1539, Aug. 1997.

[14] E. Zwicker and H. Fastl, Psychoacoustic, Facts and Models. New York: Springer-Verlag, 1990

[15] Z. Cvetković and M. Vetterli, "Tight Weyl-Heisenberg frames in $l^{2}(Z)$," IEEE Trans. Signal Processing, vol. 46, pp. 1256-1259, May 1998

[16] S. Weiß, L. Lampe, and R. W. Stewart, "Efficient subband adaptive filtering with oversampled GDFT filter banks," in Dig. IEE Colloq. Adapt. Signal Processing for Mobile Commun. Syst., no. 383, Oct. 1997, pp. $4 / 1-4 / 9$

[17] M. Harteneck and R. W. Stewart, "An oversampled filter bank with different analysis and synthesis filters for the use with adaptive filters," in Proc. Asilomar Conf. Signals, Systems and Computers, Nov. 1997, vol. 2, pp. 1274-1278.

[18] M. Harteneck, J. M. Páez-Borrallo, and R. W. Stewart, "An oversampled subband adaptive filter without cross adaptive filters," Signal Processing, vol. 64, no. 1, pp. 93-101, Jan. 1998.

[19] M. Harteneck, "Real valued filter banks for subband adaptive filters," Ph.D. dissertation, Univ. of Strathclyde, Glasgow, U.K., Apr. 1998

[20] I. Daubechies, Ten Lectures on Wavelets. Philadelphia, PA: SIAM, 1992.

[21] H. Bölcskei, F. Hlawatsch, and H. G. Feichtinger, "Frame-theoretic analysis of oversampled filter banks," IEEE Trans. Signal Processing, vol. 46, pp. 3256-3268, Dec. 1998.

[22] M. Rossi, J.-Y. Zhang, and W. Steenaart, "A new algorithm for designing prototype filters for M-band pseudo QMF banks," in Proc. EUSIPCO, Sept. 1996, vol. II, pp. 1195-1198.

[23] H. Xu, W.-S. Lu, and A. Antoniou, "Efficient iterative design method for cosine-modulated QMF banks," IEEE Trans. Signal Processing, vol. 44, pp. 1657-1668, July 1996.

[24] G. Strang and T. Nguyen, Wavelets and Filter Banks. Cambridge, MA: Cambridge Univ. Press, 1996.

[25] T. Q. Nguyen, "Near-perfect-reconstruction pseudo-QMF banks," IEEE Trans. Signal Processing, vol. 42, pp. 65-76, Jan. 1994.

[26] M. Rossi, J.-Y. Zhang, and W. Steenaart, "Iterative least squares design of perfect reconstruction QMF banks," in Proc. CCECE, May 1996, pp. 726-765.

[27] G. H. Golub and C. F. Van Loan, Matrix Computations. Baltimore, MD: The Johns Hopkins Press, 1989.

[28] S. Weiss and R. W. Stewart, On Adaptive Filtering in Oversampled Subbands. Aachen, Germany: Shaker Verlag, 1998.

[29] S. Weiss, R. W. Stewart, A. Stenger, and R. Rabenstein, "Performance limitations of subband adaptive filters," in Proc. EUSIPCO, Sept. 1998, vol. III, pp. 1245-1248. 